\title{
El impacto antrópico sobre la vid silvestre en el territorio de Lapurdi (suroeste de Francia)
}

\section{Anthropic impact on the wild grapevine in the Lapurdi region (south-western France)}

\author{
Álvaro Rodríguez ${ }^{1}$, Juanjo Hidalgo ${ }^{2}$, Jesús Arrimadas ${ }^{3}$, Carlos Alvar-Ocete ${ }^{4}$, \\ Frédéric Duhart ${ }^{5}$, Rafael Ocete ${ }^{4}$, María José Iriarte-Chiapusso ${ }^{6}$, José Manuel Valle ${ }^{1}$
}

\section{*}

\section{Resumen}

En este trabajo se hace una introducción al único taxón euroasiático de vid silvestre, Vitis vinifera L. subsp. sylvestris (Gmelin) Hegi, al proceso de su domesticación y a las causas antrópicas que han mermado drásticamente las poblaciones de esta subespecie, desde la Península Ibérica hasta Afganistán. Asimismo, se hace referencia al problema de la pérdida de biodiversidad del viñedo y se subraya la importancia de estas parras autóctonas para mitigar esa erosión genética.

En el mismo se señala que, pese a la ley de protección de este parental de las viníferas cultivadas en Francia, se ha producido una importante pérdida de ejemplares en esta zona. Según nuestras observaciones, comenzadas en 1992 y contrastadas con las de 2015, algunas zonas cubiertas con vegetación clímax atlántica han sido completamente destruidas en las inmediaciones de St. Jean de Luz, concretamente en la zona de Acotz-Chibau.

Por otra parte, se incluye la información cartográfica de las poblaciones relictas de vid silvestre que aún se conservan, con el fin de facilitar las tareas de conservación in situ de las mismas.

\footnotetext{
1 Universidad del País Vasco / Euskal Herriko Unibertsitatea (UPV/EHU) Escuela Universitaria de Ingeniería. Vitoria-Gasteiz.Idgp@ehu.eus

2 Aunia Kultura Elkartea, Luiaondo.

3 Exdirector de las Escuelas Oficiales de Idiomas del País Vasco.

4 Universidad de Sevilla. Laboratonio de Entomología Aplicada.

5 Facultad de Ciencias Gastronómicas de la Mondragon Unibersitatea.

6 Universidad del País Vasco / Euskal Herriko Unibertsitatea (UPV/EHU) IKERBASQUE. Facultad de Letras. Vitoria-Gasteiz.
} 
También se recogen datos sobre la vegetación acompañante y el estado sanitario actual de las parras.

Palabras clave: Conservación, destrucción de hábitats naturales, recurso fitogenético, Vitis vinifera L. subsp. sylvestris (Gmelin) Hegi, Lapurdi (suroeste de Francia).

\begin{abstract}
This paper introduces the only Euriasian taxon of the wild grapevine, Vitis vinifera L. subsp. sylvestris (Gmelin) Hegi, its domestication and the anthropic causes that have drastically reduced the populations of this subspecies from the Iberian Peninsula to Afghanistan. Besides, reference is made to the problem of the loss of biodiversity in the vineyard and to the importance of this native vine in order to reduce that genetic erosion.

On the other hand, it is pointed out that, although this parental of the cultured grapevine is protected by law in France, a major loss of specimens has occurred in this region. According to our observations, which began in 1992 and were verified in 2015, several areas covered with Atlantic climax vegetation were completely destroyed near St. Jean de Luz, specifically, in the Acotz-Chibau area.

Cartographic information concerning the remaining relict populations of wild grapevines is included in order to facilitate in situ conservation work. Furthermore, data on the accompanying vegetation and the health status of the grapevines are also presented.
\end{abstract}

Key words: Preservation, destruction of natural habitats, plant genetic resources, Vitis vinifera L. subsp. sylvestris (Gmelin) Hegi, Lapurdi (south west of France).

\title{
Laburpena
}

Lan honek sarrera egiten dio Eurasian dagoen basamahatsetako taxon bakarrari, Vitis vinifera L. subsp. sylvestris (Gmelin) Hegi subespeziekoari hain zuzen ere, bere etxekotze prozesuari eta bai gizakiaren eraginez subespezie horren populazioek jasandako murrizketa handiari ere, Iberiar Penintsulatik Afganistaneraino.

Halaber, mahastien biodibertsitatearen galeraren arazoari egiten zaio erreferentzia, tokian tokiko mahats-parren garrantzia azpimarratuz galtze genetiko hori ekiditeko.

Lanean aipatzen da ezen, nahiz eta Frantzian landatutako mahats-guraso hori babesteko legea indarrean izan den, ale galera garrantzitsua jazo dela eremu horretan. Gure behaketen arabera, 1992an hasitakoak eta 2015ekoekin alderatuta, atlantiar erako klimax-landaredidun eremu batzuk guztiz hondatu dira Donibane Lohizuneko inguruetan, zehazki, Akotze-Xibauko aldean.

Bestalde, oraindik erlikia moduan mantentzen diren basamahatsen populazioei buruzko informazio kartografikoa sartu da, horien kontserbazio-lanak tokian-tokian errazteko asmoz. Landaredi laguntzailearen eta mahatsondoen egungo osasun-egoerari buruzko datuak ere sartu dira.

Gako hitzak: Kontserbazioa, habitat naturalen suntsiketa, baliabide fitogenetikoa, Vitis vinifera L. subsp. sylvestris (Gmelin) Hegi, Lapurdi (Frantziako hego-mendebaldea). 


\section{Introducción}

En el presente artículo se exponen los resultados de la variación observada en el número de ejemplares de vid silvestre en el territorio de Lapurdi (Labourd, Francia) entre 1992 y 2015, así como aspectos relativos a su estado sanitario y vegetativo.

En un primer subapartado de esta introducción se esboza la diversidad de las vides silvestres propias de diferentes regiones del planeta. Por otra parte, se recogen algunos nombres con los que aparece en la literatura la vid silvestre euroasiática y los propios del País Vasco. Finalmente, se hace una sinopsis del proceso de domesticación de la citada subespecie que ha desembocado en la gran diversidad de variedades de cultivo existentes en las zonas vitícolas.

\section{La diversidad del genero Vitis L.}

En Eurasia, la única vinífera existente entre la Península Ibérica y el macizo del Hindu Kush corresponde al taxón Vitis vinifera L. subsp. sylvestris (Gmelin) Hegi, que también se encuentra en algunas zonas del Maghreb. Esta subespecie, que ha sobrevivido a los periodos glaciares del Cuaternario, constituye el parental dioico de las variedades de cultivo, que son generalmente hermafroditas dentro de Europa (Fig. 1). No obstante, en el sur del Cáucaso, que constituyó el principal refugio de muchos parentales de los frutales que fueron posteriormente aprovechados por el hombre, se conservan muchas variedades cultivadas con flor femenina (Ocete et al., 2012).

En otras zonas geográficas, como es el caso de Norteamérica y el este de China, existe un mayor número de especies silvestres autóctonas de vid. Una explicación de ello sería que los diversos periodos glaciares a lo largo del Cuaternario influyeron en la distribución de

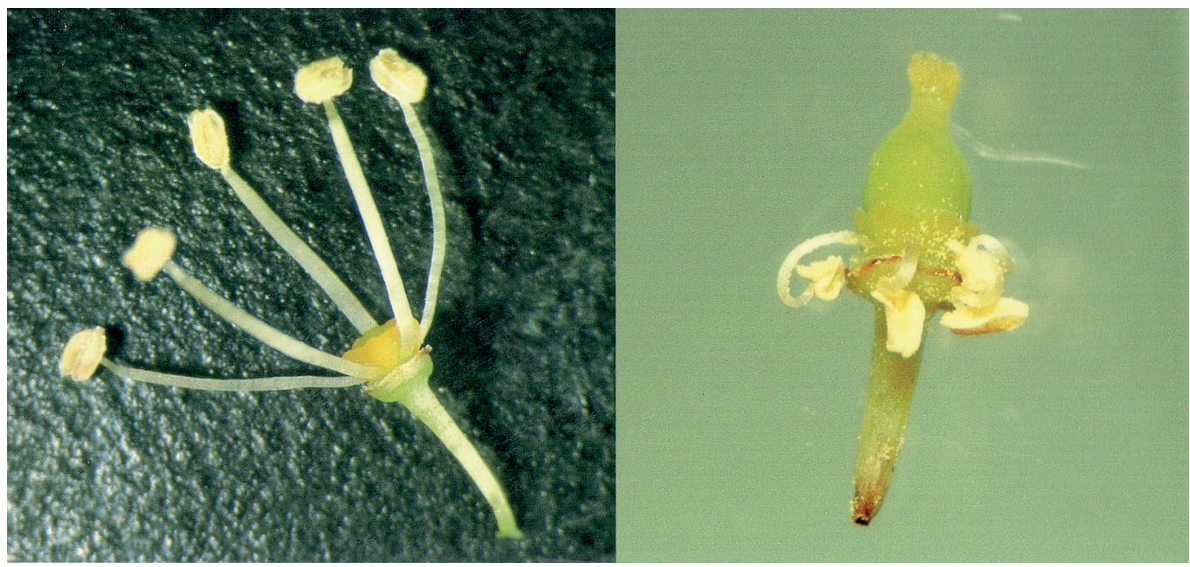

Fig. 1.- Flor masculina pura (izquierda) y flor femenina con estambres reflejos (derecha).

Fig. 1.- Male flower (left) and female flower with reflexed stamens (right). 
las comunidades vegetales y en la formación de especies. En el caso del género Vitis L., tanto en el caso de Estados Unidos de Norteamérica como en la parte más oriental de China, estas vitáceas pudieron dirigirse hacia latitudes más meridionales. Esta migración fue posible gracias a que en la zona americana las cordilleras tienen una orientación NorteSur, mientras en el este de China el relieve es poco marcado. Por eso, en la actualidad se pueden encontrar varias decenas de especies diferentes en ambas zonas geográficas (Zohary \& Spiegel-Roy, 1975). En total existen cerca de 70 especies dentro del género Vitis L.

En el resto del continente euroasiático, las diversas cordilleras tienen una orientación dominante Este-Oeste. Debido a ello, se restringió su capacidad de migración en los periodos glaciares, manteniéndose una única especie, Vitis vinifera L., que se refugió principalmente en el sur del Cáucaso (Transcaucasia), y en el sur de la cuenca mediterránea (Huglin, 1986). Las especies norteamericanas V. rupestris, V. riparia y V. berlandieri han sido las más empleadas en calidad de porta-injertos, con el fin de poder recuperar los viñedos europeos después de la invasión de la filoxera. Todas las especies de vid tienen 38 cromosomas y son interfértiles; gracias a ello, se han obtenido portainjertos por hibridación de vides americanas con europeas, que mejoran la compatibilidad con la vinífera. También, se han obtenido híbridos productores directos, muy empleados en zonas húmedas, por su resistencia al mildiu. Éstos han persistido durante varias décadas en zonas productoras de chacolí de Hegoalde y viñedos de Azores y Madeira para la producción del vinho cheiro o morangueiro.

\section{Referencias en la literatura clásica y nombres comunes de la vid silvestre}

Las cepas femeninas de la vid silvestre fueron ya citadas por Théophrasto (siglo III a.C.) como Agria ampelos, en su obra De Historia Plantarum. El célebre médico Dioscórides (siglo I), de la época de Nerón, habla de estos dos géneros, en su tratado Materia médica, de esta manera:

"Una que jamás perfecciona sus racimos, pero que los deja siempre en flor, cuyo fruto es llamado enanthe (flor de vino). Y la otra, que acaba su maduración produciendo pequeñas bayas, negras y con sabor astringente."

Posteriormente, esta vinífera silvestre fue bautizada como labrusca por Virgilio en sus Églogas (S.I. a. J.C.). Dicho nombre ha quedado registrado en español, portugués e italiano. En francés se conserva con la grafía de labrusque. En la Comunidad Autónoma Vasca se conoce con los nombres castellanos de vid silvestre, uvillas o uva de zorra. También, de forma mucho más restringida, bajo los nombres eusquéricos de basamahatsondoa o zozo mahatsa.

\section{Usos tradicionales de la vid silvestre}

En cuanto a los usos antiguos de esta vinífera, seguidamente recogemos algunos de los más usuales, según Ocete et al. (2011a) y en las referencias contenidas en dicho artículo. Las bayas de las parras silvestres fueron empleadas como alimento por los humanos en di- 
versas épocas, desde el Paleolítico (Rivera \& Walker, 1989). Lógicamente fueron la materia prima de las primeras vinificaciones. De hecho, la vinificación de los racimos silvestres se ha conservado en algunas zonas europeas hasta hace unas décadas.

En la obra de Carbonell (1820) puede leerse la siguiente reflexión:

"... es la fabricación de licores en la que el hombre ha desarrollado todo su ingenio, pues excepto el agua y la leche, todo el resto es el fruto de su obra. La naturaleza jamás ha creado licores espirituosos, no ha hecho más que favorecer la putrefacción del racimo en la cepa, mientras que su jugo se convierte gracias a este arte en un licor agradable, tónico y nutritivo, al que llamamos vino.

Es difícil indicar la época en la que el hombre comenzó a fabricarlo. Este precioso descubrimiento parece perderse en la noche de los tiempos; de tal forma que la invención del vino tiene sus fábulas, como todos los objetos cuya utilidad se ha generalizado."

Además, en algunas regiones de Italia y de Alemania, las cepas silvestres se mezclaban con las cepas cultivadas con el fin de bajar el precio de producción de los vinos caseros (Anzani et al., 1993). En Cerdeña, hasta hace unas décadas, dada la abundante conservación de poblaciones de vid silvestre en bosques de ribera, se elaboraban los vinos llamados vinu de marxani o vino de caprai (Lovicu et al., 2009). En esta misma isla, que perteneció a la Corona de Aragón, La Carta de Logu castigaba la venta de uva silvestre. Se trata de un códice de leyes, rescatado por Lovicu (2007), emanado del Giudici Arborea, contra el comercio de vid silvestre en Cerdeña. Esta prohibición estaba motivada porque este tipo de cosecha estaba incontrolado. No pagaba impuestos a las autoridades civiles y tampoco diezmos y primicias a la Iglesia. El capítulo CXLII contiene literalmente lo siguiente:

"A quien venda uva silvestre.

Item ordenamos: que si se sorprende a una persona vendiendo uva silvestre, o robada o cogida de una viña que no sea suya, pague una multa propiamente la persona que la vende. Y el vendedor que la lleve a su casa sin autorización del dueño sea encontrado en casa o en cualquier parte en posesión de dicha mercancía pagará una multa. Si no la pudiera pagar quedará en prisión por desobediencia a nuestra voluntad."

Las uvas de la vid silvestre son astringentes, y sus racimos y sus flores se mezclaban en el mosto para dar mayor duración al vino y también para darle cierto sabor rasposo, agradable al gusto, y reconfortante para el estómago. La aportación del fruto silvestre disminuía el pH del mosto puesto que aumentaba el contenido de los ácidos y facilitaba así una buena conservación del vino, mientras que los volátiles contenidos en las flores conferían a la mezcla un aroma afrutado (Ocete et al., 2011a).

Los vinos tintos provenientes de cepas silvestres maduras presentan un buen equilibrio entre el grado alcohólico y la acidez total y, después de un largo proceso de maduración, 
adquieren un bonito color (color rojo bien marcado), con una dotación polifénólica elevada que mejora la conservación del vino de base y aporta una concentración elevada de antocianinas (Ocete et al., 2007). El grado alcohólico varía entre 8 y 14,5 \% en volumen: el valor más bajo corresponde a los Pirineos Navarros y el más elevado a la provincia de Cáceres. El pH oscila entre 2,1 y 3,5 y la acidez total entre 4 y $5 \mathrm{~g}$ de ácido sulfúrico, debido a los elevados contenidos de ácido tartárico y málico, muy superiores a los de las viníferas tradicionales cultivadas.

La intensidad del color es un parámetro sin unidades, obtenido por la adición de las absorbancias a 420, 520 y $620 \mathrm{~nm}$. Dicho valor en la Península Ibérica oscilaba entre 11,1 y 26,4 . Este rango indica que el vino es "es de buena capa" (vino tinto bien marcado).

En lo referente a la totalidad de polifenoles, la cifra media si sitúa alrededor de 80 mg/l, lo que indica que se trata de vinos con dotación polifenólica elevada, como para recibir un tratamiento prolongado. Por otra parte, la concentración de antocianinas por encima de $300 \mathrm{mg} / \mathrm{l}$ indica que la uva silvestre tiene una poderosa capacidad de aportar color, con una buena estructura polifenólica (Ocete et al., 2004).

Hasta hace unos 25 años, se ha producido vinagre casero en las estribaciones de la Serranía de Grazalema (Cádiz) y en algunas aldeas de Sierra Morena (Jaén), dentro de Andalucía. Ha de tenerse en cuenta que este derivado del vino ha sido, junto a la sal marina extraída de los yacimientos del Trias de facies germánica (Keuper), un importantísimo conservante alimentario. También el vinagre ha sido empleado como mejorante de las pastas cerámicas, ya que evita la aparición de fisuras durante la cocción de las piezas (Carreño, 1995).

El vino, en general, siempre ha tenido una aureola medicinal. Si observamos, por ejemplo, la antología de canciones populares vascas de ambos lados de los Pirineos (Hidalgo \& Llano, 2007):

Arno xuri, arno gorri

arno kolore ederra

mundu huntan dan argelenari

hik emaiten dakok indarra.

Vino blanco, vino tinto

vino de hermoso color

al más débil de este mundo

le otorgas vigor

Arno hunik balin bada

ez akhar niri barberik.

Si hay buen vino

no me traigan barbero (médico o cirujano),

como se cantaba en el pueblo de Amikuze (Basse-Navarre). 
Medicu, barbero gustiac

Erremedie aundiac

Dituzta besterentzat

Baña beti berenzat

Escatzen dute lenena

Ardo zarric dan onena

Los médicos y los cirujanos / grandes remedios / conocen / cuando sufren los otros / pero para ellos / lo que primero piden / es el mejor de los vinos viejos

La farmacopea está llena de remedios medicinales que tienen su origen en la vid silvestre, principalmente en la savia, hojas y agraz. En cuanto a los usos medicinales de las vides silvestres, existen varias referencias en los distintos textos españoles antiguos recogidos por Font Quer (1999).

Laguna (1570) ensalzó las propiedades medicinales de las parras silvestres, principalmente de los ejemplares masculinos:

"... la hemos olvidado hasta tal punto que no nos debe nada, pero aún se esfuerza en servirnos y en agradarnos con lo que puede; quiero decir que su fruto, con sus taIlos, con sus hojas y con sus flores y sus utilizaciones infinitas, principalmente cuando está madura y que cubre perfectamente un miembro. Estas facultades son mucho más eficaces en la labrusca y principalmente en la que produce el enanthe que en la vid cultivada..."

Por su parte, Quer (1784) indicaba:

"De las vides silvestre y cultivada se han hecho distintos usos en Medicina. El agua que pierde o llora en primavera es aperitiva, diurética y buena para los ojos, y es para estas enfermedades que algunos las conservan."

En la provincia de Burgos (Castilla y León) según la Noticia histórica corográfica del Muy Noble y Real Valle de Mena, datada de 1796, se dice:

"Hay muchas vides en los montes y en el borde de los caminos y en las riberas de los ríos y su fruto es muy bueno para el agraz."

Los racimos de uva, silvestre o cultivada fueron una ofrenda mortuoria muy generalizada entre diversas culturas de la Península Ibérica, desde la Cultura argárica (Edad del Bronce) hasta la Paleocristiana. La morfología de pepitas encontradas en las tumbas nos indica, por tanto, si pertenecían a parras silvestres o cultivadas.

En Grecia, la mitología recogida por Eurípides, según la cual Dionisio acompañado por su séquito de Ménades llegó a Tebas con la intención de establecer allí su culto. Plantó viñas 
alrededor de la tumba de su madre, Sémele, fulminada por la visión de Zeus. Penteo, hijo de Ágave, hermana de Sémele, y rey de Tebas, que no creía en su divinidad, prohibió su culto. Cadmo era el abuelo de Penteo y Dionisio, padre de Sémele y de Ágave, antiguo rey de Tebas. En la tragedia Las Bacantes (Eurípides, siglos V y IV a.C.) se puede leer:

"Veo cerca del palacio la tumba de mi madre, la fulminada, y los escombros de su morada, humeantes del fuego de Zeus, atestando para siempre la venganza de Hera, su insulto a mi madre.

Agradezco a Cadmo haber hecho este suelo inviolable para los pasos humanos, el santuario de su hija. He querido extender, todo alrededor, una verde red de sarmientos adornados de racimos."

Ovidio (siglo I a.C.) indicaba:

"Es también el momento de honrar las tumbas, de apaciguar las almas de los antepasados y de aportar pequeñas ofrendas sobre los montículos de tierra de las sepulturas. Los Manes piden pocas cosas (...), se contentan con el don de las coronas que recubren una teja, de algunos granos (de uva), de una pizca de sal, de pan empapado en vino y violetas dispersas."

En algunas estelas y tapas de cistas funerarias de época romana con epigrafía vasca, halladas en la zona del Haute-Garonne aparecen frecuentemente racimos de uva (Fig. 2).

Las parras silvestres han sido utilizadas, además, como portainjertos naturales, muy bien adaptados al terreno, como en el caso de la Península Balcánica (Zimmermann, 1958).

Los sarmientos se han empleado para la fabricación de maromas (Quer, 1784) y aros para la construcción de nasas de pesca en el litoral asturiano y gaditano hasta hace dos décadas.

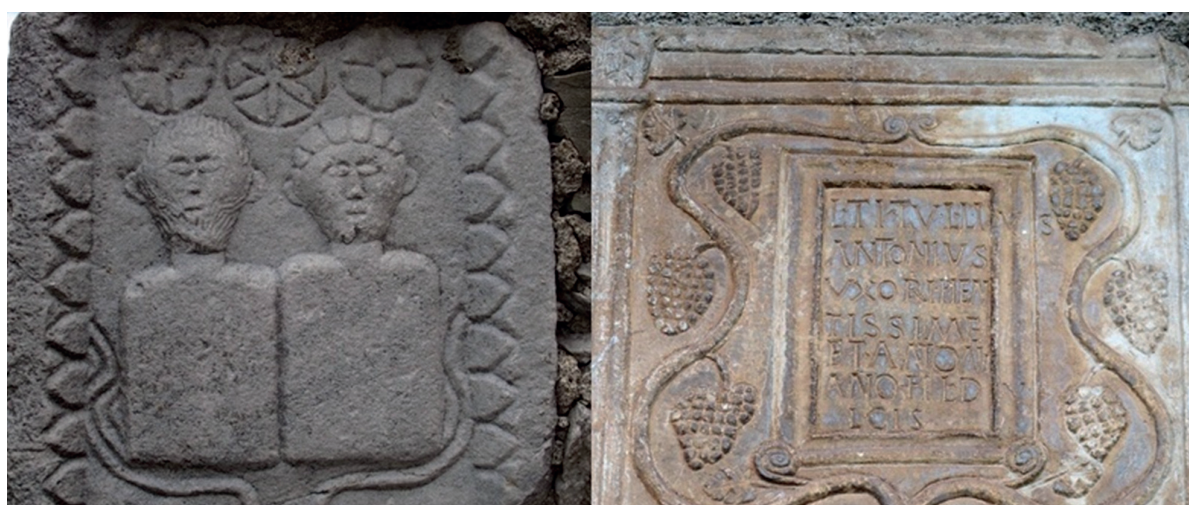

Fig. 2.- Iconografía de racimos en lápidas de la época romana: Chapelle Saint-Julien, Saléchan (Hautes-Pyrénées) y église de Billière (Haute-Garonne).

Fig. 2.- Iconography of bunches on gravestones from the Roman era: Chapelle Saint-Julien, Saléchan (Hautes-Pyrénées) and Billière church (Haute-Garonne). 


\section{El proceso de domesticación y la importancia de la vid silvestre como recurso fitoge- nético}

La domesticación de la vid se basa en la selección humana de ejemplares silvestres hermafroditas autofecundables (Forni, 2006), procedentes de una mutación de parras con flor masculina. Al poseer flor hermafrodita se las encuadra dentro de la subespecie Vitis vinifera L. subsp. sativa DC. ex Hegi, de la que existen, actualmente varios miles de variedades.

Parece ser que aunque el centro principal de domesticación se encuentra en el Sur del Cáucaso (Ramishvili, 2001), dentro del llamado Triángulo de la Uva Fértil de Vavilov, los microsatélites de ADN cloroplástico parecen indicar que existen otros centros secundarios de domesticación. En las variedades cultivadas y parras silvestres del sur del Cáucaso, en el caso de la Península Ibérica, el clorotipo A es el propio de todas las parras silvestres analizadas y de la mayor parte de las cepas cultivadas consideradas autóctonas. Este clorotipo es, también, el más extendido en el resto de las viníferas de Europa occidental (Arroyo-García et al., 2006).

La Viticultura constituye una de las manifestaciones más importantes de la milenaria cultura mediterránea. Las primeras semillas con morfología cultivada datan de unos 6000 años a.C. y fueron halladas en la República de Georgia, en el yacimiento de Shulaveri Gora (Mc Govern, 2003; Ramishvili, 2001).

Queremos subrayar, en este sentido, las palabras de este gran maestro de maestros, Alain Huetz de Lemps, Profesor emérito de la Universidad de Burdeos, recogidas en el prólogo de la obra de Ocete et al. (2007):

"Los historiadores y los geógrafos se interesan muy particularmente por el origen de las plantas cultivadas y quieren saber cómo se produce el paso de la planta silvestre a la planta cultivada. En lo referente a la vid euroasiática, la Vitis vinifera, existe, al mismo tiempo, una subespecie llamada también lambrusca, y una subespecie cultivada comprende cientos de variedades."

Ahondando en la información del párrafo anterior, debe decirse que las parras silvestres constituyen un importante recurso fitogenético, ya que se encuentran libres de la selección humana, dirigida hacia una mejor adaptación al cultivo, aumento de la producción, mayor contenido de azúcares en el fruto, aumento del tamaño de la baya, coloración, etc. (Forni, 2006).

Entre las características más destacables de los ejemplares femeninos silvestres se encuentran la elevada acidez y elevada intensidad de color de sus mostos, incluso en zonas de clima mediterráneo. Ambos factores son de gran importancia a la hora de emprender programas de mejora genética de variedades tintas de cultivo, máxime dentro del actual contexto de calentamiento global. Por otra parte, muestran una gran resistencia al encharcamiento y, en muchos casos, a la caliza activa. Éstas son dos importantes propiedades para la obtención de nuevos portainjertos (Ocete et al, 2007).

El viñedo se encuentra sometido a una importante y creciente erosión genética. Los viñedos han pasado de tener distintas variedades-población a simplemente clones, reducién- 
dose gravemente la agrodiversidad, es decir, la base genética sobre la que actúa la selección natural. Ello constituye un grave problema de cara al cambio de las condiciones climatológicas y a la aparición de nuevos agentes causantes de estrés biótico: virus, fitoplasmas, bacterias, hongos y plagas, principalmente de artrópodos (Ocete et al., 2007). Por lo tanto, se debe salvaguardar toda la biodiversidad de las cepas silvestres, que como ya se ha comentado, albergan un importante pool genético (Myles et al., 2011).

Tampoco deben perderse las antiguas variedades tradicionales, generalmente, muy escasas en los actuales viñedos de producción. En la gran mayoría de las zonas vitícolas se ha producido una importante reducción del número de vidueño de cada Denominación de Origen. Y en varias de ellas se han introducido variedades internacionales, generalmente de origen francés. Por ejemplo, en Andalucía, estamos asistiendo atónitos a una expansión de la variedad Shyrah, que produce vinos tintos con buena coloración bajo clima cálido (Ocete et al., 2007).

Por último, hay que destacar que la viña constituye el cultivo más extendido y de mayor rendimiento económico del planeta (Vivier \& Pretorius, 2002).

\section{Causas de la regresión de las poblaciones de vid silvestre}

El impacto antrópico tiene diversas formas de actuar, como ha quedado muy bien reflejado en Arnold (2002). Antes de la llegada de los citados parásitos americanos, las parras silvestres parece que eran bastante comunes en Eurasia, por la bibliografía existente y la cantidad de usos de las parras. Lógicamente, la llegada del oídio y mildiu sería la responsable de la muerte de los ejemplares más sensibles de cada población. También la introducción de plantas invasoras norteamericanas, como portainjertos e híbridos productores directos, para la reconstrucción del viñedo filoxerado, ha hecho que, en muchos casos, compitan con nuestras parras silvestres, desplazándolas de sus hábitats naturales. Dichas viníferas autóctonas son mucho más resistentes y tolerantes a las enfermedades criptogámicas de origen norteamericano (Ocete et al., 2007).

Por otra parte, las riberas de los ríos son suelos fértiles que pueden dedicarse a diversos usos hortícolas y explotaciones forestales. En ellas se llegan a introducir árboles exóticos de rápido desarrollo para su explotación maderera, como los eucaliptos de Galicia, Asturias, Cantabria, País Vasco, Extremadura y Andalucía. Hay que añadir la transformación y limpieza de los cursos de agua, en los cuales se arrasa con la vegetación riparia. También, la modernización de la red viaria y la construcción de zonas de ocio y el uso de desbrozadoras y herbicidas para mantener limpios sus márgenes (Arnold, 2002).

Debe apuntillarse, que no sólo desaparecen las parras, paralelamente, lo hace también la biocenosis de artrópodos auxiliares que regulan las poblaciones de fitófagos que viven sobre las mismas. La diversidad y abundancia de éstos es mucho más compleja que la encontrada sobre las parras cultivadas, debido a las operaciones de manejo del viñedo, donde influye decisivamente el empleo de diferentes tipos de pesticidas (Ferragut et al., 2009). 


\section{Objetivos del estudio}

El objetivo del presente artículo es denunciar las recientes pérdidas de parras silvestres en el área indicada, pese a que Francia cuenta con una legislación específica de protección en el Decreto del 20 de enero de 1982 referente a la lista de especies vegetales protegidas en el conjunto del territorio (La versión consolidada del decreto puede encontrarse en https://www.legifrance.gouv.fr/affichTexte.do?cidTexte=JORFTEXT000000865328 [revisión sep-2016]). Al mismo tiempo, se pretende georeferenciar las poblaciones relictas, de forma que se puedan preservar con efectividad y poder analizar su evolución a lo largo del tiempo.

\section{Área de estudio y métodos}

Las primeras prospecciones de parras silvestres en Iparralde fueron realizadas por nuestro equipo de trabajo en 1987. Desde 1992 hasta 2015 inclusive, se han realizado 20 observaciones de poblaciones de vid silvestre en la zona de Lapurdi comprendida entre las localidades de Hendaya, Biarritz, St. Jean de Luz, Ghetary y Ahetze. En las mismas se ha observado el tipo de flor de las parras entre finales de junio y principios de julio, con el fin de determinar su carácter dioico y analizar el resto de características ampelográficas más importantes. También se han determinado las principales especies de la vegetación acompañante. Paralelamente, se han realizado muestreos sobre la presencia de síntomas causados por plagas y enfermedades, tanto a nivel de raíz, que fueron descubiertas hasta una profundidad de $30 \mathrm{~cm}$, como de los órganos aéreos.

Por otra parte, se ha realizado una comparación entre el número de individuos de cada población en ambos extremos del periodo de tiempo indicado, con el fin de estimar la pérdida de ejemplares.

\section{Resultados y discusión}

Las coordenadas y altitud de las 7 poblaciones principales encontradas aparecen en la Tabla 1, donde también se recogen los censos de parras de 1992 y 2015.

Según los datos expuestos se ha producido una pérdida del 43,63\% de las parras. La destrucción más reciente del hábitat natural se ha producido durante el primer trimestre de 2015 (Fig. 3), a la salida de St. Jean de Luz, donde únicamente están rebrotando 2 pequeñas parras, sujetas a las vallas de la obra, junto a la carretera D810 (Fig. 4). En la cuneta opuesta, señalada como Acotz I, ha habido un aumento de parras debido a la aparición de plántulas procedentes de semilla, que se encuentran rodeadas de especies exóticas introducidas.

Hace años que la zona de la desviación a Ahetze fue transformada en un espacio deportivo para la práctica de skate-board, donde se perdieron los 13 ejemplares indicados. Por otra parte, en los dos últimos años se ha producido una limpieza de la pequeña carretera que se encuentra por encima de la playa de Chibau hasta la calle Murillo del barrio de Acotz-Chibau. 


\begin{tabular}{|l|c|c|c|c|c|}
\hline \multirow{2}{*}{ Lugar } & Latitud (N) & Longitud (W) & $\begin{array}{c}\text { Altitud } \\
\text { media } \\
(\mathrm{m})\end{array}$ & $\begin{array}{c}\mathrm{N}^{\circ} \\
\text { Parras } \\
1992\end{array}$ & $\begin{array}{c}\mathrm{N}^{\circ} \\
\text { Parras } \\
2015\end{array}$ \\
\hline Guethary-Acotz & $43^{\circ} 24^{\prime} 56^{\prime \prime}-43^{\circ} 24^{\prime} 58^{\prime \prime}$ & $1^{\circ} 36^{\prime} 42^{\prime \prime}-1^{\circ} 36^{\prime} 44^{\prime \prime}$ & 27,5 & 20 & 12 \\
\hline Acotz (deviation a Ahetze) & $43^{\circ} 24^{\prime} 44^{\prime \prime}-43^{\circ} 24^{\prime} 45^{\prime \prime}$ & $1^{\circ} 36^{\prime} 54^{\prime \prime}-1^{\circ} 36^{\prime} 52^{\prime \prime}$ & 40,5 & 13 & 0 \\
\hline Acotz-D & $43^{\circ} 24^{\prime} 36^{\prime}-43^{\circ} 24^{\prime} 41^{\prime \prime}$ & $1^{\circ} 37^{\prime} 21^{\prime}-1^{\circ} 37^{\prime} 12^{\prime \prime}$ & 39,8 & 31 & 2 \\
\hline Acotz-1 & $43^{\circ} 24^{\prime} 33^{\prime \prime}-43^{\circ} 24^{\prime} 40^{\prime \prime}$ & $1^{\circ} 37^{\prime} 24^{\prime \prime}-1^{\circ} 37^{\prime} 12^{\prime \prime}$ & 34 & 7 & 10 \\
\hline Garbaiari (ermita) & $43^{\circ} 24^{\prime} 42^{\prime \prime}$ & $1^{\circ} 37^{\prime} 25^{\prime \prime}$ & 37 & 5 & 1 \\
\hline Plage de Chibau & $43^{\circ} 24^{\prime} 37^{\prime \prime}-43^{\circ} 24^{\prime} 40^{\prime \prime}$ & $1^{\circ} 38^{\prime} 06^{\prime \prime}-1^{\circ} 38^{\prime} 00^{\prime \prime}$ & 29 & 11 & 6 \\
\hline Sortie de Sokoa & $43^{\circ} 23^{\prime} 17^{\prime \prime}-43^{\circ} 23^{\prime} 24^{\prime \prime}$ & $1^{\circ} 41^{\prime} 59^{\prime \prime}-1^{\circ} 41^{\prime} 52^{\prime \prime}$ & 38 & 23 & 7 \\
\hline Coste & $43^{\circ} 23^{\prime} 27^{\prime \prime}$ & $1^{\circ} 42^{\prime} 13^{\prime \prime}$ & 18 & 7 & 2 \\
\hline Chemin d'Etzan & $43^{\circ} 23^{\prime} 01^{\prime \prime}-43^{\circ} 23^{\prime} 08^{\prime \prime}$ & $1^{\circ} 42^{\prime} 37^{\prime \prime}-1^{\circ} 42^{\prime} 42^{\prime \prime}$ & 26,7 & 23 & 11 \\
\hline
\end{tabular}

Tabla 1.- Localización y altitud de las poblaciones e indicación del número de ejemplares en 1992 y en 2015 (coordenadas geográficas, sistema de referencia ETRS89).

Table 1.- Location and altitude of populations and indication of the number of individuals in 1992 and 2015 (geographical coordinates, ETRS89 reference system).

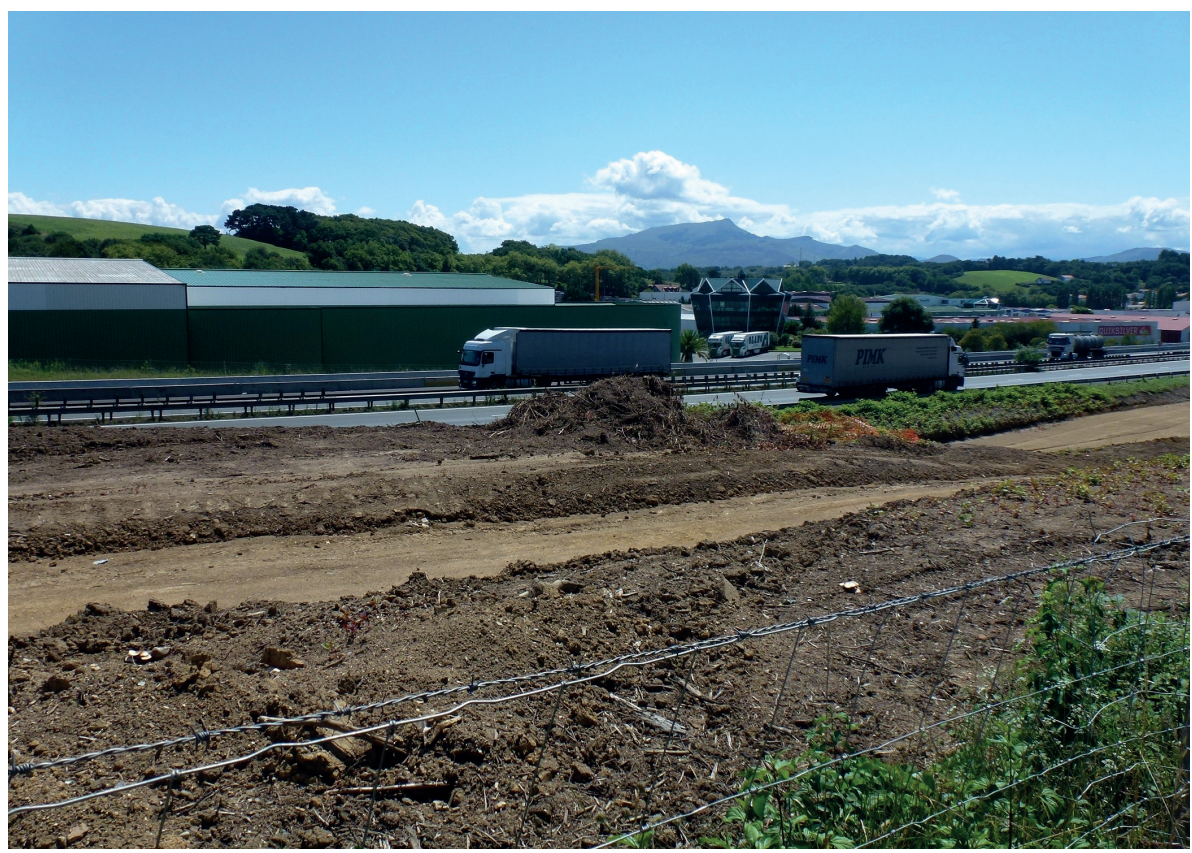

Fig. 3.- Zona de bosque devastada en el barrio de Acotz (carretera D810).

Fig. 3.- Area of devastated woodland in the Acotz area (D810 road). 


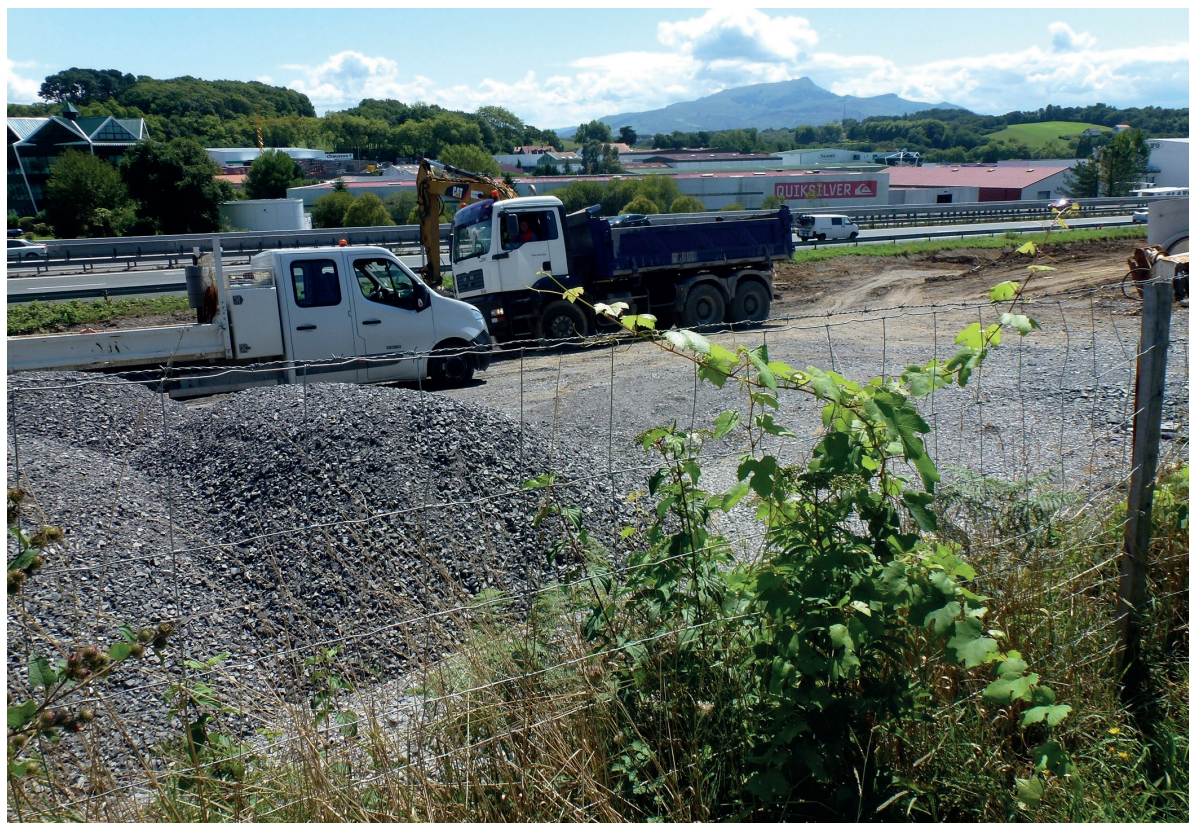

Fig. 4.- Nuevos brotes procedentes de parras cortadas en la zona de la figura anterior.

Fig. 4.- New shoots from vines cut in the area indicated in the previous figure.

\section{Hábitats y vegetación acompañante}

Las parras silvestres son unas lianas que utilizan sus zarcillos para subir por la vegetación circundante, a la manera de un tutor, y captar así una luminosidad con la intensidad adecuada en las canopias (Fig. 5).

Las principales angiospermas que suelen servir de tutor a las parras, dentro de la zona de estudio, son: Acer campestre, Acer monspessulanum, Alnus glutinosa, Corylus avellana, Crataegus monogyna, Fraxinus ornus, Laurus nobilis, Prunus spinosa, Quercus ilex, Quercus faginea, Rhamnus alaternus, Robinia pseudoacacia (introducida en las cunetas de las carreteras), Rosa sempervirens, Rubus ulmifolius, Salix atrocinerea, Salix eleagnos, Sambucus nigra, Sorbus aucuparia, Tilia cordata, y Viburnum tinus. Entre las pteridofitas, la más frecuente es Pteridium aquilinum.

Otras lianas frecuentes son: Bryonia dioica, Clematis vitalba, Hedera helix, Lonicera periclymenum y Smilax aspera.

Los componentes de la vegetación acompañante citada son típicos de la vegetación coluvial-riparia atlántica, propia de lugares que tienen temperaturas moderadas y precipitaciones cercanas a 1200 mm/año. Las series de vegetación pueden ser Polysticho setiferiFraxino excelsioris sigmetum, Hyperico androsaemi-Alno glutinosae sigmetum et Salici angustifolio-albae sigmetum, según Loidi et al. (2009). 


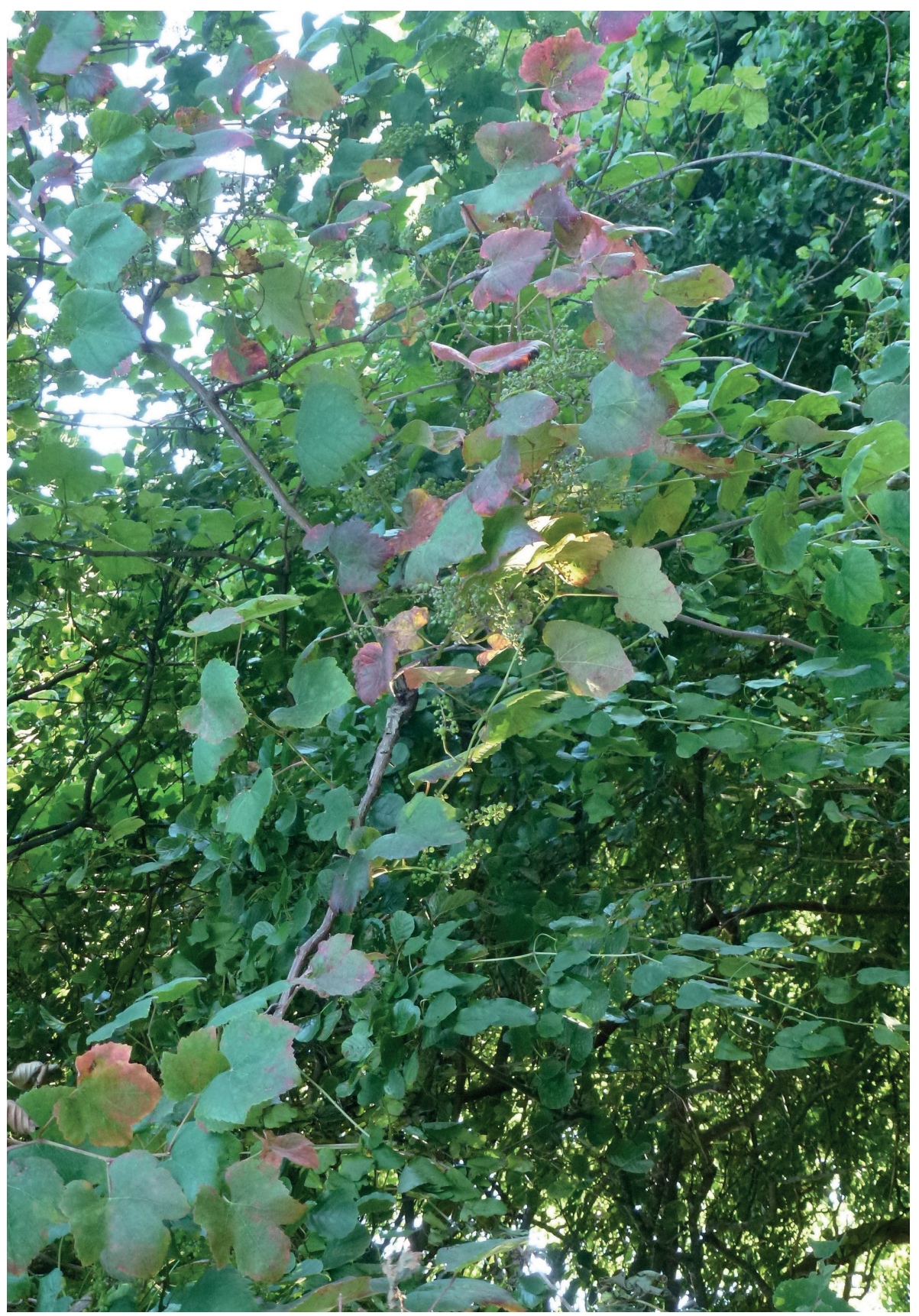

Fig. 5.-Ejemplares de vid silvestre sobre sus tutores en las inmediaciones de Sokoa.

Fig. 5.- Specimens of wild vines on their canes near Sokoa. 


\section{Descripción ampelográfica}

Las cepas masculinas tienen una flor puramente masculina, mientras que las que producen las uvas tienen la flor femenina con estambres reflejos.

Los estambres de las flores masculinas son más largos y rectos que los de las flores femeninas, que son reflejos, con polen acolporado. La misión de las primeras consiste en producir grandes masas de polen que garanticen la polinización anemógama de las flores de los ejemplares del otro sexo, razón por la que presentan granos de polen tricolporados.

La totalidad de los zarcillos son bífidos, con una distribución discontinua sobre el sarmiento en la rama. Su longitud y su solidez son muy variables según los distintos ejemplares, incluso de una misma población.

Las hojas adultas varían desde una pequeña talla a talla mediana. Generalmente el interior del peciolo de los individuos masculinos está más abierto que el de los femeninos. El color de la cara superior es con frecuencia verde claro. Existe frecuentemente una débil pigmentación rojiza antociánica en los nervios principales. Normalmente, los dientes de las plantas femeninas tienen los dos lados iguales, cóncavos o convexos; a veces, uno rectilíneo y el otro cóncavo o convexo. En los ejemplares masculinos los dientes tienen, habitualmente, los dos lados convexos o rectilíneos.

Los sarmientos presentan una sección elíptica, con la superficie lisa color marrón, de intensidad bastante variable.

Los racimos de las plantas femeninas son de pequeña talla y siempre tintas, generalmente flácidas, por falta de fecundación de algunas flores. Las bayas son de pequeño tamaño y poco uniformes, de color vino tinto. Su forma es subesférica, con un diámetro máximo de aproximadamente $1 \mathrm{~cm}$. Por lo general las semillas son bastante menos alargadas que las que provienen de las variedades cultivadas, con la chalaza más corta (Fig. 6), como aparece bien reflejado en las conclusiones sobre la morfología de la muestra de semillas de otras zonas francesas analizadas por el INRA de Montpellier (Terral et al., 2010). El número de semillas por baya varía de 1 a 3.

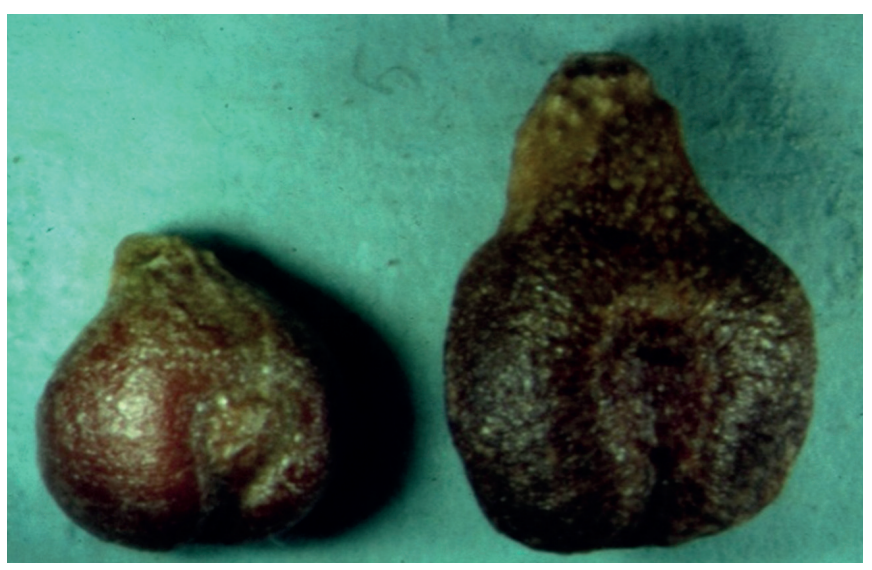

Fig. 6.- Semilla de vid silvestre (izquierda) y cultivada (derecha).

Fig. 6.- Wild vine seed (left) and cultivated vine seed (right). 


\section{Estado sanitario de las poblaciones}

Cuando se manifestaron los daños producidos por el oidio americano sobre el viñedo francés, se empezaron a importar masivamente cepas de América del Norte, buen número de ellas híbridas con viníferas europeas, que no eran sensibles al hongo. Estas plantas han servido de vector a un pulgón, llamado filoxera por Planchon, que corresponde al actual taxón Dactulosphaira vitifoliae (Fitch), (Hemiptera, Phylloxeridae). En este sentido, las palabras de De la Escosura y Deleito (1878) son muy elocuentes:

"El comercio que las naciones europeas mantienen con los Estados Unidos en lo referente a los productos vinícolas, ha sido la causa de la introducción, en los años 1858 a 1860, en Inglaterra, en Irlanda, en Francia, en Portugal, en Hungría, en Austria y en ciertos viveros de Alemania, de plantas, de sarmientos, de esquejes y de injertos de vides americanas que, a causa de su rápido crecimiento y floración, eran muy solicitadas; desde entonces, la filoxera, tan poco exigente en cuanto a las condiciones del terreno y tan terrible por su fecundidad, ya no ha abandonado Europa y el hombre, imprudentemente, se ha encargado de extenderla distribuyendo y propagando por doquier las plantas infestadas."

En la totalidad de los ejemplares examinados en el presente trabajo, las raicillas no presentan ningún síntoma provocado por la filoxera, tampoco por nematodos formadores de quistes teniendo en cuenta las condiciones edáficas de las poblaciones, establecidas en suelos húmedos que permanecen inundados varios meses al año. Sin embargo, esta subespecie de vid es sensible a la filoxera, como se ha comprobado experimentalmente en distintas poblaciones situadas en posición aluvial y coluvial desde Portugal a Hungría (Ocete et al., 2011b).

Un hecho a resaltar es que en Francia, considerando la rápida expansión de la filoxera en la segunda mitad del s. XIX, se pensó, en un principio, injertar las viníferas sobre las vides silvestres. Esta idea fue rápidamente rechazada gracias a las observaciones realizadas por Camile Saint-Pierre, que pusieron en evidencia que las cepas silvestres eran también sensibles a la fase radicícola de la plaga. En los escritos de De la Branchère (1876) encontramos lo siguiente:

"Hemos pensado injertar las vides cultivadas sobre la lambrusca o vid salvaje, tan frecuente en nuestros bosques, pero las observaciones de Camile Saint-Pierre han demostrado que la lambrusca, por símisma, no es capaz de escapar a la filoxera y que también recibe sus ataques."

En cuanto a la infestación por artrópodos fitófagos hay dos tipos de ácaros, omnipresentes en todas las poblaciones, como ocurre en la práctica totalidad de las poblaciones vascas, centroeuropeas y del sur del Cáucaso (Ocete et al. 1994, Maghradze et al. 2015).

Colomerus vitis (Pagenstecher) (Acari, Eriophyidae) es una especie monófaga ampliamente extendida en los viñedos de los dos hemisferios. Generalmente, produce erineos repartidos sobre la superficie inferior de las hojas. Calepitrimerus vitis (Nalepa) (Acari, Eriophyidae) es 
otra especie monófaga. Como en el caso anterior se encuentra presente en la mayor parte de las poblaciones indicadas en las últimas citas bibliográficas.

Con respecto a los patógenos, hay síntomas de ataques de las dos enfermedades fúngicas americanas, de oidio que aparecen frecuentemente, Erysiphe necator Schweinitz) Burril, y de mildiú, Plasmopara viticola (Berkeley \& Curtis) Berlease \& de Toni, tanto en las hojas como en los sarmientos y en los racimos. En cada población estudiada, hay que subrayar los distintos grados de susceptibilidad que muestran las distintas cepas de una misma población frente a los dos ácaros y hongos, debida probablemente a la diversidad genotípica de sus ejemplares.

Conviene resaltar que, en el momento presente, los fitófagos y los patógenos no constituyen una amenaza para las cepas silvestres de la región. Sin embargo, las enfermedades fúngicas americanas han provocado, sin duda, un impacto considerable que ha influido en la drástica reducción de sus poblaciones, como ya se ha referido anteriormente.

\section{Consideraciones finales}

Como puede concluirse, las parras silvestres están sufriendo una tremenda regresión en esta zona de Lapurdi, como ya fue denunciado por Ocete et al. (2012), a pesar de su protección legal (título modificado por el decreto del 31 de agosto de 1995). Urge tomar medidas para la conservación de este recurso fitogenético amenazado, tanto in situ como ex situ, en bancos de germoplasma. Máxime cuando existen pruebas palinológicas de su presencia en los alrededores de la actual ciudad de Biarritz desde el Pleistoceno (Olfield, 1968). Se han perdido hábitats naturales, que albergaban parras con importante bagaje genético y unas complejas condiciones ecológicas. Sirva de ejemplo que el estudio realizado con material procedente de Acotz D reveló la existencia de una interesante comunidad de micorrizas asociadas a las raíces de las parras (Ocete et al., 2015a).

La Organización Internacional de la Viña y el Vino (OIV), en su resolución 424/2010, emanada de su Asamblea General, recomienda "emprender cuanto antes amplias campañas de prospección destinadas a catalogar el material salvaje y cultivo en peligro de extinción e identificar, cuando proceda, los genotipos originales o aún no descritos ni caracterizados". La importancia de las parras silvestres como fuente de genes utilizables en programas de mejora varietal aparece recogida en el proyecto europeo GRAPEGEN 06.

Dentro del proyecto europeo COST Action FA 1003 "East-West Collaboration for Grapevine Diversity Exploration and Mobilization of Adaptative Traits for Breeding" se pretende buscar una figura de protección legal dentro de la Unión Europea (Ocete et al., 2015b)

Como medida conservacionista, en el caso de la Comunidad Autónoma Vasca, existe un banco de germoplasma en la Estación de Fruticultura de Zalla (Bizkaia), dependiente de la Diputación Foral de Bizkaia / Bizkaiko Foru Aldundia. En Álava, el Servicio de Biodiversidad de la Diputación Foral de Álava / Arabako Foru Aldundia ha empezado a realizar una reserva de estas parras en los alrededores del Lago de Arreo (Caicedo Yuso). Éstas podrían ser unas iniciativas aplicables al caso de Lapurdi. 


\section{Bibliografía}

- Anzani, R., Failla, O., Scienza, A., de Micheli, L. 1993. Individuazione e conservacione del germoplasma di vite selvatica (Vitis vinifera silvestris) in Italia. Vignevigne 6: 51-60.

- Arnold, C. 2002. Écologie de la vigne sauvage en Europe (Vitis vinifera ssp. silvestris). Geobotanica helvetica 76: 1-276.

- Arroyo-García, R., Ruiz-García L., Bolling, L., Ocete, R., López, M.A., Arnold, C., Ergul, A., Söylemezo, G., Lu, H., Uzun, I., Cabello, F., Ibáñez, J., Aradhya, M.K., Atanassov, A., Atanassov, I., Balint, S., J.L. Cenis J.L., Costantini, L., Gorislavets, S., Grando, M.S., Klein, B.Y., Mc Govern, P.E., Merdinoglu, D. Pejic, I., Pelsy, F., Primikirios, N., Risovannaya, V., Roubelakis-Angelakis, K.A., Snoussi, H., Sotiri, P., Tamhankar, S., This, P., Troshin, L., Malpica, J.M., Lefort, F. \& Martinez-Zapater, J.M. 2006. Multiple origins of cultivated grapevine (Vitis vinifera L. ssp. sativa) based on chloroplast DNA polymorphismes. Molecular Ecology 15(12): 3707-14.

- Carbonell, F. 1820. Arte de hacery conservar el vino. Imp. Antonio Brusi. Barcelona.

- Carreño, E. 1995. Las poblaciones de vid silvestre de la Península Ibérica y el origen de las variedades actuales. Rev. Murciana de Antropología 12 : 35-44.

- De La Blanchére, H. 1876. Les ravageurs des Vergers et des Vignes. J. Rothschild Editeur. París.

- De la Escosura, L. y Deleito, V. 1878. La Phylloxèra. Descripción, vida y costumbres. Establecimiento Tipográfico de A. Bacaycoa. Madrid.

- Ferragut, A. Gallardo, Ocete, R., López, M.A. 2009. Natural predatory enemies of the Erineum strain of Colomerus vitis (Pagenstecher) (Acari, Eriophyidae) found on wild grapevine popula tions from southern Spain (Andalusia). Vitis 47: 51-54.

• Font Quer, P. 1999. Plantas medicinales, el Dioscórides renovado. Ed. Lábor. Barcelona.

- Forni, G. 2006. Dall'origine della viticoltura alla sua introduzione in Italia. In: La vite e I'uomo. II Ed. F. Del Zan, O. Failla \& A. Scienza (Eds.) Ersa. Gorizia.

• Hidalgo, J., Llano, J.M. 2007. Chacolí de Álava. Ed. J.M. Llano. Bilbao.

- Huglin, P. 1986. Biologie et ecologie de la vigne. Ed. Payot. Lausanne.

- Laguna, A. 1570. Pedazio Dioscórides Anazarbeo, acerca de la materia medicinal y de los venenos mortíferos. Salamanca.

- Loidi, J., Biurrun, I., Campos, J.A., García-Mijangos, J.A., Herrera, M. 2009. La vegetación de la Comunidad Autónoma del Páis Vasco. Gobierno Vasco. Vitoria-Gasteiz. Disponible en: http://www.ingurumena.ejgv.euskadi.eus/contenidos/documentacion/series_vegetacion/es _def/adjuntos/Vegetacion_CAPV.pdf.

- Lovicu, G. 2007. La vite selvatica e i vittigni autoctoni della Sardegna: testimoni silenziosi (e poco conosciuti) de una viticultura antigua. Darwin Quaderni 7: 7478.

- Lovicu, G., Farci, M., Bacchetta, G., Orrú, M., Pérez, M.A., Gómez, J., Ocete, R. 2009. Hábitats, estado sanitario y caracterización enológica de la vid silvestre, Vitis vinifera L. spp. sylvestris (Gmelin) Hegi, en Cerdeña (Insula vini). Enólogos 62: 30-35. 
- Maghradze, D., Salimov, V., Melyan, G., Musayev, M., Ocete C.A., Chipashvilli, R., Failla, O., Ocete, R. 2015. Sanitary status of the Eurasian wild grapevine in the South Caucasian region. Vitis 54: 203-205.

- Mc Govern, P.E. 2003. Ancient Wine: The Search for the origins of Viniculture. Princeton University.

- Myles, S., Biykob, A.R, Owense, C.R., Browna, P.J., Grassi, P., Aradhya, M.K. 2011. Genetic structure and domestication history of the grape.

Disponible en: www.pnas.org/cgi/doi/10.1073./pnas.1009363108.

- Ocete, R., Armendáriz, I., Cantos, M., Álvarez, D., Azcón, R. 2015a. Ecological characterization of wild grapevine habitats focused on arbuscular mycorrhizal symbiosis. Vitis 54: 207-211.

- Ocete, R., Arnold, C., Failla, O., Lovicu, G., Biagini, B., Imazio, S., Lara, M., Maghradze, D., Lopez, M.A. 2011 b. Consideration on European wild grapevine (Vitis vinifera L., ssp. sylvestris (Gmelin) Hegi) and Phylloxera infestation. Vitis 50(2): 97-98.

- Ocete, R., Arrimadas, J., Hidalgo, J. 2012. La vigne sauvage dans le Pays Basque, une ressource phytogénétique en danger en Memoria Satului Românesc. Banat Village Museum. Editorial Brumar. Timisoara.

- Ocete, R., Cantos, M., López, M.A., Gallardo, A., Pérez, M.A., Troncoso, A., Lara, M., Failla, O., Ferragut, F. J., Liñán, J. 2007. Caracterización y conservación del recurso fitogenético vid silvestre en Andalucía. Ed. Consejería de Medio Ambiente, Junta de Andalucía. Sevilla.

- Ocete, R., Gallardo, A., Pérez, M.A., Ocete, C., Lara, M., López, M.A. 2011 a. Usages traditionnels de la vigne sauvage en Espagne. Territoires du vin [en ligne], 2011: Les territoires du vin en Espagne, 22 février 2011. Disponible sur Internet: http://revuesshs.u-bourgogne.fr/territoiresduvin/document.php?id=872 ISSN 1760-5296.

- Ocete, R., Lara, M. 1994. Consideraciones sobre la ausencia de síntomas de ataque por filoxera en poblaciones autóctonas de Vitis vinífera silvestris (Gmelin) Hegi. Bol. San. Veg. Plagas 20(3): 631-636.

- Ocete, R., López, M.A., Gallardo, A., Arnold, C., Pérez, M.A., Rubio, I. 2004. Euskal herriko eta inguruetako basamahatsandoa: ekologia, kokapena eta arriskuak. Servicio Central de Publicaciones del Gobierno Vasco. Vitoria-Gasteiz.

- Ocete, R., Fevereiro, P., Failla, O. 2015b. Proposal for the wild grapevine (Vitis vinifera L. subsp. sylvestris (Gmelin) Hegi) conservation in the European countries. Vitis 55: 281-282.

- Oldfield, F. 1968. The Quaternary vegetational history of the French Pays-Basque. I. Stratigraphy and polen analysis. New phytologist 67: 677-731.

- Quer, J. 1784. Continuación de la Flora Española ó Historia de las plantas de España. Joaquín Ibarra. Impresor de Cámara de S.M. Madrid.

- Ramishvili R. 2001. History of Georgian Grape and Wine. Tbilisi.

- Rivera, D., Walker, M.J. 1989. A review of paleobotanical findings of early Vitis in the Mediterranean and on the origin of cultivated grape-vines, with special reference to new pointers to prehistoric explotation in the Western Mediterranean. Rev. Paleobotany 6: 205-237. 
- Terral, J.F., Tabard, E, Bouby, L., Ivorra, S, Pastor, T., Figueiral, I., Picq, S., Chevance, J.B., Jung, C., Fabre, L., Tardy, C., Compan, M, Bacilieri, R., Lacombe, T., This, P. 2010. Evolution and history of grapevine (Vitis vinifera) under domestication: new morphometric perspectives to understand seed domestication syndrome and reveal origins of ancient European cultivars. Annals of Botany 105: 443-455.

- Vavilov, N.I. 1926. Cemtry proiskhozhdenia kulturnikh rastenii (The centres of origin for cultivated plants). Trudi poprikladnoi botanike, genetike i selektsii (Proceedings of Applied Botany, Genetics and Breeding) 16: 133-137.

- Vivier, V.A., Pretorius, I.S. 2002. Genetically taibored grapevines for the wine industry. Trends Botechnology 20: 472-488.

- Zimmermann, J. 1958. Die Bedeutung der Wildrebe (Vitis silvestris Gmel.) in Jugoslavien für Forschung und Weinbau. Wein-Wiss 8: 79-87.

- Zohary, D., Spiegel-Roy, P. 1975. Beginnings of fruit growing in the Old World. Science 187: 319-327.

- Fecha de recepción/Date of reception: 21-04-2016

- Fecha de aceptación/Date of acceptance: 02-10-2016

Editor Asociado/Associate editor: Ricardo Ibáñez 